\title{
An Image Segmentation Algorithm Based on Gray Bit Plane for Flame
}

\author{
Xing Deng ${ }^{1}$, Jinlan $\mathrm{Li}^{2}$, Fujian Feng ${ }^{2}$, Lin Wang $^{2}$, Zhongming $\mathrm{Yu}^{2,3}$ \\ 1 School of physics and electronic science, Guizhou Normal University, Guiyang, P.R.China, \\ 550001 \\ 2 Guizhou provincial key laboratory of pattern recognition and intelligent system, Guiyang, \\ P.R.China, 550025 \\ 3 Liupanshui Normal University, Liupanshui, P.R.China,553000 \\ (E-mail:794682477@qq.com, yzm69333@163.com,470202030@qq.com,wanglin@gznc.edu.cn)
}

Keywords: Fire detect; OHTA color space; gray bit plane; image segmentation

\begin{abstract}
Flame region judgment is an important part of fire detection system; meanwhile the flame of intelligent monitoring system also has important practical significance to national fire control safety. Therefore, this article puts forward the flame image segmentation method based on gray level bit plane. Firstly, by studying the distribution of flame image in OHTA color space, we found that it can accurately segment the flame area by using the method of the gray level bit plane for component image in OHTA color space. The experiments results show that the algorithm in different scenarios for segmenting flame has a good effectiveness and robustness.
\end{abstract}

\section{Introduction}

Light and heat from the burning of fire is material, is a form of energy.Must have fuel, ignition and combustion gas oxygen (not necessarily) to burn, the landscapes themselves.Since 2000, our country each year about 40000, a fire killed more than 2000 people, disabled people, 3000-4000 fire caused by the direct property losses every year more than 10 , one hundred million yuan.Thus property losses and casualties caused by fire is immeasurable.Therefore, how to quickly and accurately identify the location of the point of ignition becomes our urgent problem.

The traditional fire detection is mainly based on sensor of automatic fire alarm technology.Mainly according to the detection of smoke generated when the fire broke out, the parameters such as temperature and light, after signal processing, compare, judge the signal fire alarm.but fire detection based on close, however, because of distance, fire signal will weaken, misstatement, omission of fire situation is serious.

In recent years, along with the computer image processing technology matures, fire detection based on computer vision technology has become a hot research topic.Because objects around the flame color has obvious visual characteristics, played a key role in the process of flame recognition, Therefore, many scholars put forward different flame color detection algorithm.Jessica Ebert and Jennie Shipley using the characteristics of the flame in RGB space through the experiment to determine a R, G, B component scope to analyze and extract the flame,T.Celiks determined in HSI space H, S, I each component is a scope to this intersection to extract the flame.

The above algorithm, the detected flame zone caused miscarriage of justice likely affected scenes, lighting conditions and similar flame color interference and the like. Therefore, this article analyzes the flame image in OHTA color space distribution of grayscale bit plane method OHTA color space component of image segmentation, experimental results show that: the algorithm under different scenarios flame dividing good and effective and robustness

\section{OHTA Color Space}

OHTA on the basis of the lessons learned, For eight different colors of the image area dividing 
System Experiment, We get a very effective color features, And summarized into a set of orthogonal color feature set $\left\{I_{1}, I_{2}, I_{3}\right\}$, We call OHTA color space, Which is defined in two ways, one definition of which is as follows:

$$
\left\{\begin{array}{l}
I_{1}=(R+G+B) / 3 \\
I_{2}=(R-B) / 2 \\
I_{3}=(2 G-R-B) / 4
\end{array}\right.
$$

Another is defined as follows:

$$
\left\{\begin{array}{l}
I_{2}^{\prime}=R-B \\
I_{3}^{\prime}=(2 G-R-B) / 2
\end{array}\right.
$$

Compared with the traditional HIS, HSV color space, color space conversion between OHTA and RBG color space is linear, it does not occur when using a non-linear color space appear (such as HSI, HSV, etc.) and the RGB color space conversion color singularity. And OHTA space each component computing simple and independent. So widely used in color image segmentation.

In order to better illustrate OHTA component color space suitable for the flame divider, I select images of different scenes to experiment, and lists the S component and OHTA RGB color space R component and HIS color space in FIG. 1 components of the image color space.
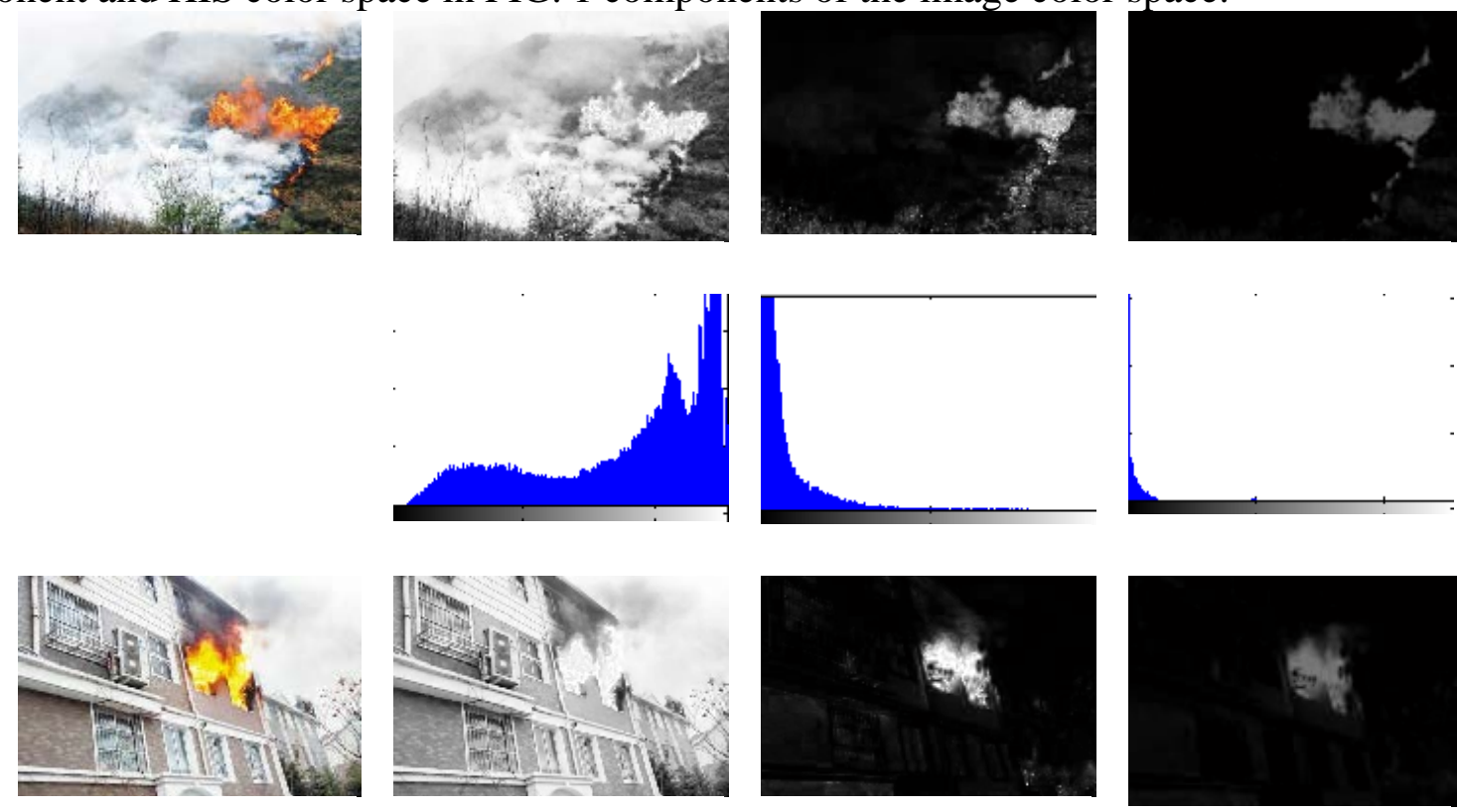



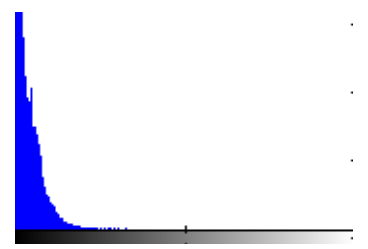

(c)

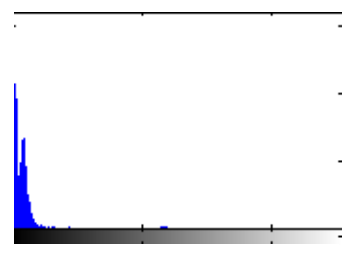

(d) (b)

(a)

Fig. 1 Each component

(a) as the original image of RGB space, (b) as the R component and histogram, (c) HSI space S component diagram and its histogram, (d) As OHTA space I2 component diagram and its histogram.

First, FIG. 1 (b), (c), (d) comparing the flame zone column, we can see (c) columns and (d) column of flame image area exhibits a high luminance value, but (c) the non-listed flame zone some 
luminance values close to the flame region luminance value, is not conducive to accurate segmentation of the flame zone. Secondly, according to the histogram (b) we can see a list of images in complex environments in the image histogram mostly multimodal, even find the optimal threshold segmentation, segmentation of the target region will have a number of different gray levels the target area. And (d) column we see OHTA color space component of the image histogram is unimodal or bimodal characteristics, so further illustrates the components of the image OHTA color space more suitable flame segmentation.

\section{Flame-bit gray-plane model}

The grayscale each pixel is only a sampling of the color image, and usually appears as gray from black to white, which ranges matrix element is generally $[0,255]$. So its data type is 8-bit unsigned integer (int8), which is often referred to all grayscale images. Grayscale image with 256 gray levels This feature explains how the wealth of information contained in the gray image, the gray-scale image information can reflect the level of darkness in each pixel. We can get what we want or video images via mobile phones, cameras, camcorders and other devices, save formats are generally RGB color model.

This paper selects the grayscale color space like OHTA component diagram, OHTA color space is converted to an RGB space. Only get a grayscale image in order to carry out further research on grayscale images. Depending on the division of gray levels, the image may show different information, which may not reflect the overall grayscale display of information, which is gray bit plane.

Construction gradation bit planes specific steps:

(1) obtain each frame image maximum grayscale value max. Max the maximum grayscale value is converted to a binary number, and calculate the number of binary digits n. (Image maximum gray value of 255, it is)

(2) to convert the pixel values of each pixel in the image is n-bit binary. Then there are n-bit gray-scale corresponding plan.

(3) for each pixel value corresponding to n-bit binary number, from right to left, taking the corresponding number of the first bit plane $f_{1}(i, j)$, the second bit plane $f_{2}(i, j)$, the third plane $f_{3}(i, j)$, Until the n-bit bit plane.

Following the 7-bit grayscale image of a plan view of the visible flame.

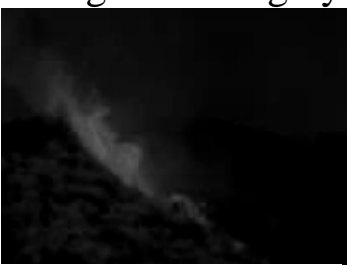

component grayscale of 12

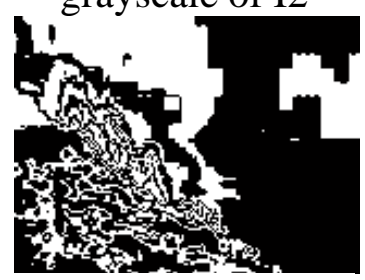

The fourth bitmap

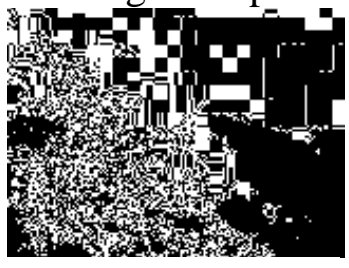

The first bitmap

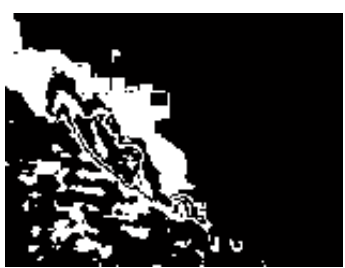

The fifth bitmap

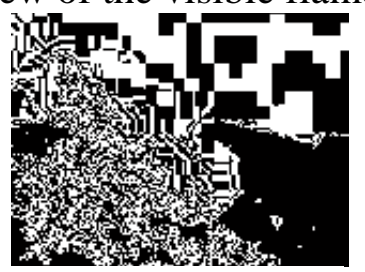

The second bitmap

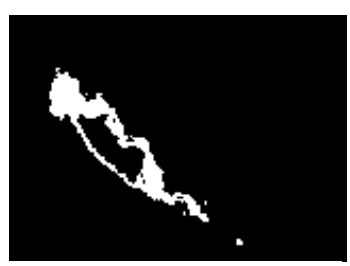

The sixth bitmap

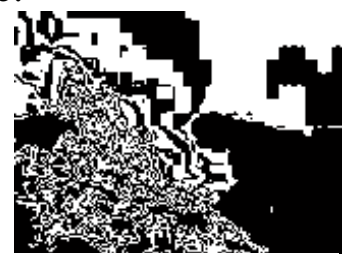

The third bitmap

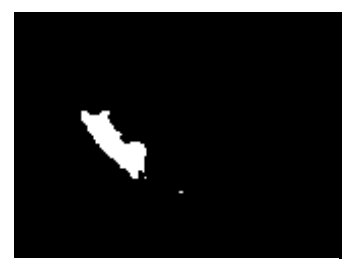

The Seventh

bitmap

Fig. 2 Bit grayscale plan 


\section{Flame zone segmentation based on gray-scale bit-plane}

In order to obtain high image information, We need to stabilize or cut the low information smoothed high preprocess information measures to preprocess. In this paper, we will use the Mean filter on the gray-scale image processing color space OHTA. Mean filter is a typical linear filtering algorithm, the template is the target pixel centered around eight pixels a filter template, then the average of all the pixels in the template to replace the original pixel value. The advantage is that the image blurring and noise reduction. Its template:

$$
w=\frac{1}{9}\left[\begin{array}{lll}
1 & 1 & 1 \\
1 & 1 & 1 \\
1 & 1 & 1
\end{array}\right]
$$

Filtered image is as follows:

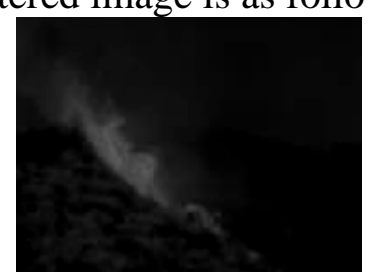

I2 Component

grayscale

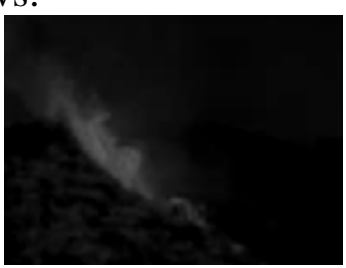

Grayscale images smoothed

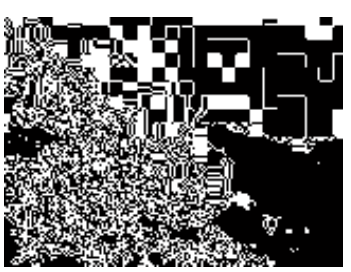

the first bitmap smoothed

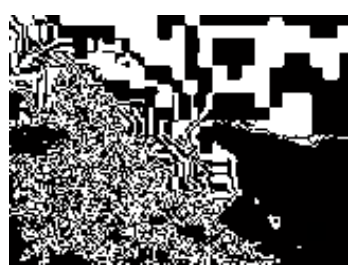

the second bitmap smoothed

Fig. 3 Mean smoothing filter

Since the mean filter in the image denoising while undermining the details of the image, which for high-bit grayscale bitmap plane plane information extraction is more appropriate, integrated processing and analysis above, this paper proposes a method of dividing the flame zone.

$$
F(i, j)=f_{n}(i, j)+f_{n-1}(i, j)
$$

Therefore, according to the equation (5) can calculate a more complete segmentation of the flame zone.

\section{Experimental results and analysis}

The experiment was carried out mainly in MATLAB7.5 simulate, Since OHTA color space ${ }_{2}$ component of the overall image is dark, and the brightness information is relatively small, so gray bit plane corresponding to the number of bits is uncertain, the highest level possible for 7 or 8 or even 6. Therefore, this paper is the second most significant bit and bit-plane for the upper and to obtain the flame zone. The results shown in Figure 4:
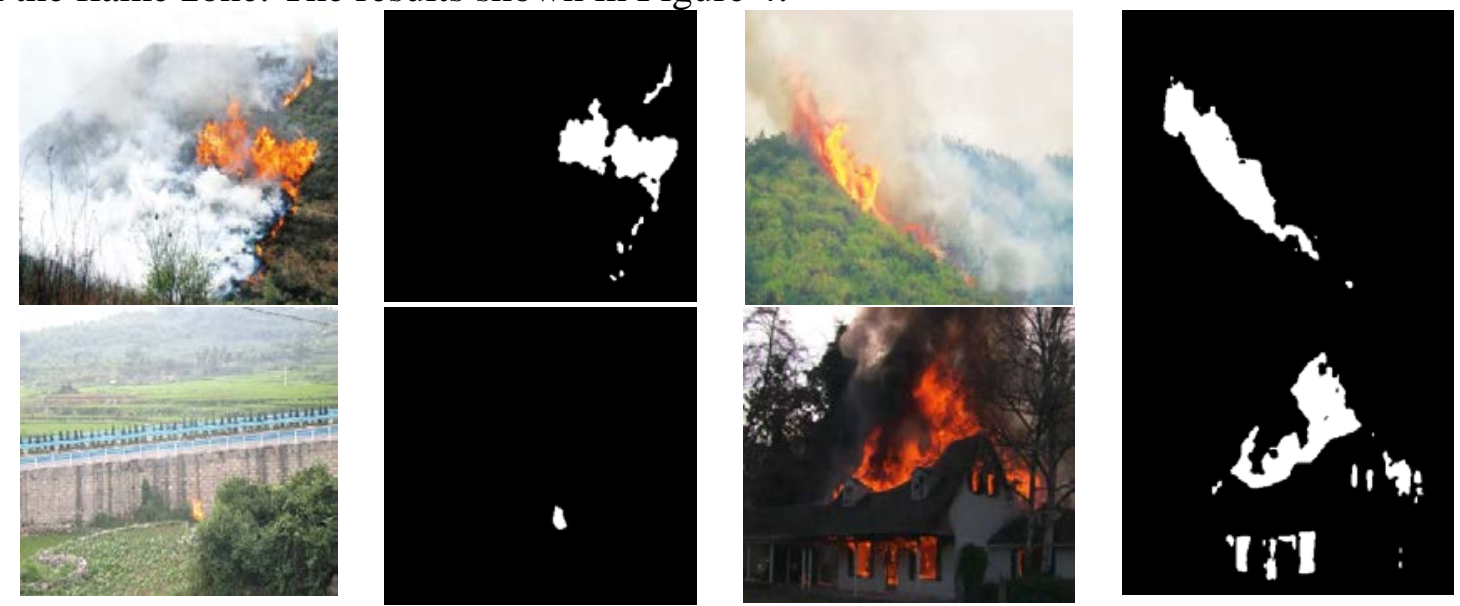


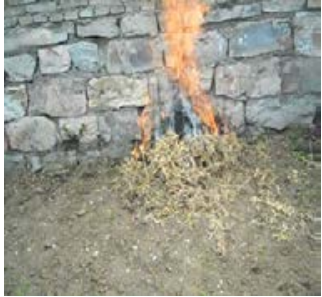

(a)

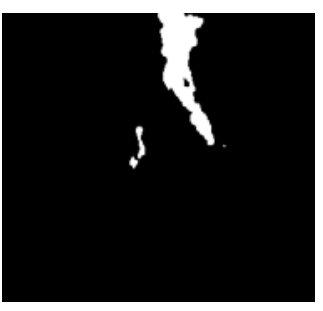

(b)

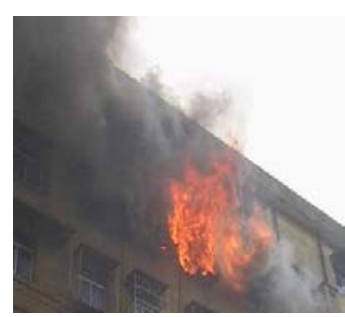

(c)

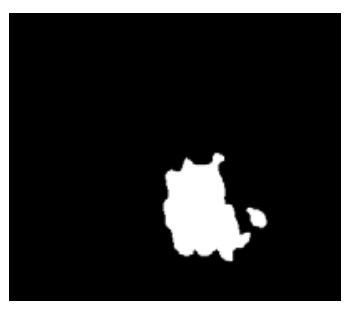

(d)

Fig. 4 Images segmentation based on gray bit planes

\section{Conclusions}

Firstly, convert the image from the RGB color space to OHTA color space, and then use the template OHTA mean filtering component of the image color space smoothing, to reduce low-bit-plane information, and enhance the accuracy of the high bit-plane information. Finally, the gray bit-plane image segmentation algorithm, the final extract a relatively complete flame zone. Through a lot of experiments in different scenarios to illustrate the feasibility of the proposed method can not only accurately, effectively extract flame zone, split time and average about 0.4 seconds. This increases the detection rate and reduce the false alarm rate, while the most important is to have a good applicability.

\section{Acknowledgments}

This work was financially supported .by the science and technology foundation of GuiZhou Province(Qian scientific cooperation J word LKLS[2013]No.01) fund

\section{References}

[1]Wang.W.H,L.H,.2011. Research of Key Technologies of Fire recognition based on Video sequences[J].Computer Simulation,28:304-307

[2]Ko.S.J, Lee S H, Lee K H. 1998. Digital Image Stabilizing Algorithms Based on Bit-Plane Matching [J].IEEE Trans Consumer Electronics, 44:617-622.

[3]Ohta Yu-Ichi, Kanade Takeo.1980. Color Information for Region Segmentation [J]. Computer Graphics and Image Processing, 13:222-241.

[4]T. Celik, H. Demirel, H.Ozkaramanli, M.Uygu. 2007. Fire Detection Using Statistical Color Model in Video Sequences [C]. ICASSP, 18:176-185. 